\title{
A multivariate comparison of two land-surface models integrated into an Arctic Regional Glimate System model
}

\author{
Amanda Lynch, ${ }^{1 *}$ David McGinnis, ${ }^{2}$ William L. Chapman, ${ }^{3}$ Jeffrey S. Tilley ${ }^{4}$ \\ ${ }^{1}$ Antarctic CRC, University of Tasmania, Hobart 7001, Tasmania, Australia \\ ${ }^{2}$ CIRES, University of Colorado, Boulder, CO 80309-0449, U.S.A. \\ ${ }^{3}$ Department of Atmospheric Sciences, University of Illinois, Urbana-Champaign, IL 61801, U.S.A. \\ ${ }^{4}$ Geophysical Institute, University of Alaska-Fairbanks, Fairbanks, AK 99775-7320, U.S.A.
}

\begin{abstract}
Different vegetation models impact the atmospheric response of a regional climate model in different ways, and hence have an impact upon the ability of that model to match an observed climatology. Using a multivariate principal-component analysis, we investigate the relationships between several land-surface models (BATS, LSM) coupled to a regional climate model, and observed climate parameters over the North Slope of Alaska. In this application, annual cycle simulations at $20 \mathrm{~km}$ spatial resolution are compared with European Centre for Medium-Range Weather Forecasts (ECMWF) climatology. Initial results demonstrate broad agreement between all models; however, small-scale regional variations between land-surface models indicate the strengths and weaknesses of the land-surface treatments in a climate system model. Specifically, we found that the greater surface-moisture availability and temperature-dependent albedo formulation of the LSM model allow for a higher proportion of low-level cloud, and a later, more rapid transition from the winter to the summer regime. Crucial to this transition is the seasonal cycle of incoming solar radiation. These preliminary results indicate the importance of the land-surface hydrologic cycle in modelling the seasonal transitions.
\end{abstract}

\section{INTRODUCTION}

The complex heterogeneity of the land surface, its associated ecosystems and its interactions with atmospheric processes are important factors in determining the role and significance of land-surface exchange processes in global climate. The role of these systems is particularly important in the context of climate change, since these processes concern not only exchanges of energy and moisture, but also exchanges of greenhouse gases such as $\mathrm{CO}_{2}$ (carbon dioxide) and methane. The high-latitude land surface is particularly sensitive, due to the presence of permafrost, and the importance of snow and ice in the intra- and inter-annual variability of the system. Further, recent analysis of observations in the Arctic regions suggests a warming of several degrees over land areas (Chapman and Walsh, 1993), as well as regional variations in sea ice and snow cover (Maslanik and others, 1996). The mean energy-flux change required to produce these observed temperature changes is estimated to be $0.6-0.7 \mathrm{Wm}^{-2}$ (Osterkamp and others, 1994). In the continuous permafrost zone, this warming will affect lake and coastal process, eolian activity and ecosystem dynamics, and eventually could produce changes in the active-layer depth. In the discontinuous permafrost, which is within a few degrees of thawing, changes in active-layer depth would occur much more rapidly. In fact, in some sites, thawing

* Present address: CIRES, University of Colorado, Boulder, CO 80309-0216, U.S.A. from both the top and the bottom has already been observed (Osterkamp and Lachenbruch, 1990). The depth of the active layer is a major determinant of the vegetative and hydrologic characteristics of northern land areas. In addition, these changes in permafrost distribution will have far-reaching effects on the global climate system due to their role in carbon storage. Thus, it is crucial to characterize the physical processes taking place in the Arctic land-surface climate system.

Land-surface processes are highly dependent upon scale. While general circulation model (GCM) performance for the polar regions has improved dramatically in recent years (e.g. Bromwich and others, 1995), they are still restricted by computational resources to very broad spatial scales. Our approach uses a limited-area climate-system model to investigate the role of land-surface processes at high resolutions. The Arctic Region Climate System Model (ARCSyM) (Lynch and others, 1995) has been developed for the study of land-ice-atmosphere and ocean-ice-atmosphere interactions in the western Arctic. This model has been used in concert with two land-surface exchange parameterizations to determine the ways in which different vegetation models will impact atmospheric components of a regional climate model, and how these have an impact on the skill of the model to match observed atmospheric conditions.

In this paper our purpose is to determine the impacts of two land-surface models on the atmospheric-boundary layer using several methods. First, we assess the general performance of the ARCSyM simulations against European Centre for Medium-Range Weather Forecasts (ECMWF) 
analyses. Prior model performance assessments indicate that model output should be broadly similar to the ECMWF analyses. A multivariate analysis is also used to investigate the spatio-temporal characteristics of model performance and, finally, specific station data are used to provide detailed model-performance analyses.

\section{METHODOLOGY}

Annual cycle simulations were performed with the ARCSyM for the year 1992, using 12 hourly ECMWF analyses to provide the boundary and initial conditions. Results from the first six months of these simulations (the spring transition) are discussed here. The experimental domain is the North Slope of Alaska (Fig. 1) with a $45 \times 75$ model grid, at $20 \mathrm{~km}$ horizontal resolution, with 23 sigma levels in the vertical. The ARCSyM is based upon the NCAR regional climate model RegCM2 (Giorgi and others, 1993), and includes the NCAR Community Climate Model Version 2 (CCM2) radiative-transfer scheme. Non-convective, resolvable-scale moist processes are modelled using prognostic equations for cloud water, rain water and water vapor in super-freezing conditions, and for cloud ice, snow and ice crystals in sub-freezing conditions. Convective processes are represented by two steady-state circulations (updraft and downdraft) with environmental mixing at the base and top of the cloud. In these experiments, the sea-surface temperature and sea ice are constrained, based on sea-surface temperatures from Shea and others (1992) and SSM/I data respectively, with the non-land surface-energy balance being performed using the Parkinson and Washington (1979) thermodynamic scheme.

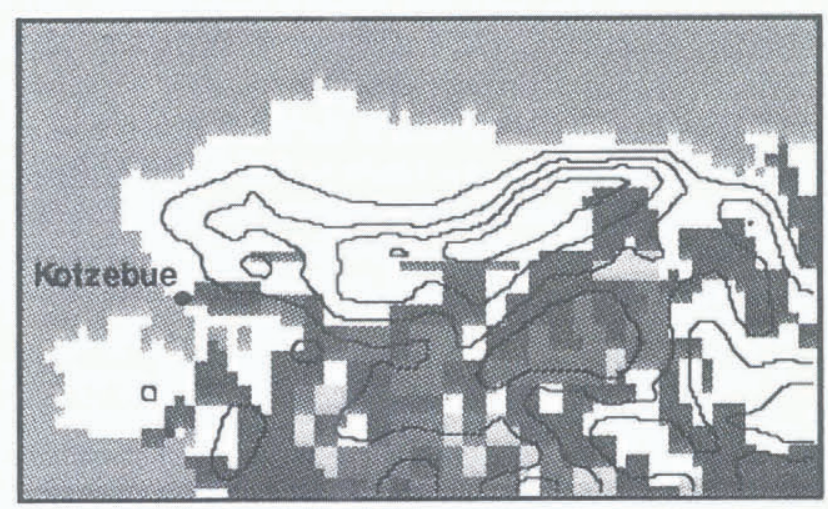

Fig. 1. ARCSy M computational domain showing the northern half of Alaska, with topography (contours) and vegetation (shading). The lightest shade indicates tundra, the darker shades indicate evergreen and deciduous forest.

Two land-surface vegetation models were incorporated within the ARCSyM regional climate model: (i) the Biosphere-Atmosphere Transfer Scheme (BATS) (Dickinson and others, 1992), widely used in GCMs as well as some regional climate and weather-prediction models; and (ii) the NCAR Land-Surface Model (LSM) (Bonan, 1995). BATS is a sophisticated land-surface vegetation model with multiple soil layers, a simple soil hydrology, and a complex vegetation treatment. BATS allows one vegetation type for each gridcell, as well as bare ground and snow cover. However, the model was not designed for, nor extensively tested in, the Arctic tundra regions. LSM is similar in many respects to the BATS model formulation, but includes improvements such as delayed infiltration and $\mathrm{CO}_{2}$ exchange processes, as well as increased soil layers for both soil hydrology and thermodynamic exchange. Further, the LSM model allows up to three vegetation types for each gridcell, as well as lakeand marsh-surface types, bare ground, and snow cover. While BATS parameterizes snow albedo using zenith angle and a snow-age parameter, LSM uses a parameterization that depends on zenith angle, soot content and snow-grain radius. A temperature dependence to the snow-grain radius causes the albedo to decrease as snow-melt begins.

The ECMWF analyses and the output from the ARCSyM simulations with each land-surface model were placed on the same grid (the $45 \times 75$ model grid), and monthly means were calculated. The atmospheric variables surface atmospheric temperature (SFT), surface mixing ratio (SFQ and sea-level pressure (SLP) provide the comparison variables to assess the basic performance of the model, and the impact of different land-surface treatments. SLP is calculated from surface pressure using the hypsometric equation and a standard lapse rate of $6.5^{\circ} \mathrm{C} \mathrm{km}^{-1}$. While this may introduce some discrepancies due to the frequent occurrence of inversions, consistency between the datasets is maintained by using the same conversion process in each case.

Principal component analysis (PCA) is a commonly used statistical procedure to disaggregate complex datasets into a smaller number of multivariate relationships. A matrix is constructed with six monthly mean values (January-June) of SLP, SFT, and SFQ (columns), with the land gridpoints (2016) forming the rows (after McGinnis and Crane, 1994). We impose a two-gridcell exclusion around the outer boundary of the domain to minimize effects of the boundary conditions in the model. In this PCA configuration, the procedure extracts a number of pseudo-variables from the correlation matrix of the variables' six monthly means. All data columns are standardized within the PCA to avoid problems associated with the vastly different magnitudes of the climate variables. Two eigenvector components are retained based on rule N (Overland and Priesendorfer, 1982) that are linear combinations of the original variables and represent abstractions that describe patterns of coherent variance (both spatially and temporally) in the correlation matrix. Varimax rotation maximizes the explained variance of each eigenvector. In this analysis, the eigenvector loadings form a time series depicting the relative correlation of each monthly variable (SLP, $\mathrm{SFT}, \mathrm{SFQ}$ to the eigenvector. These time series demonstrate a multivariate seasonality of the data. The PCA scores represent the spatial manifestation of the eigenvector where high (low) values are found in gridcells with strongly similar (opposite) time series as the eigenvector. We note that this procedure is different from many climatological uses of PCA; the most common use of PCA is to examine the spatial variance of a single climate parameter over time. Using our original data-matrix configuration, the present PCA has the benefit of allowing analysis of both spatial and temporal multivariate variance.

\section{RESULTS}

The traditional technique for comparing the performance of two model realizations is to compare particular quanti- 
ties from the simulations with an observational analysis. For example, using this technique we show the SFT biases of the BATS and LSM realizations compared to the ECMWF observational analysis for January 1992 (Fig. 2). The BATS winter simulation is generally much colder than the L.SM simulation, with larger negative biases when compared to the observational analyses over much of the domain. The deviations are largest over the land-surface area, particularly the higher elevations and the tundra regions. The LSM simulation is considerably warmer, reducing the biases substantially over the land regions, although in the lower-lying areas south of the Brooks Range this general warmth leads to small positive discrepancies.

While examining such biases can be a good guide as to the general performance of the model, it is a limited and univariate approach. Because of the large amounts of output available from any model simulation, it is advantageous to use a data-reduction technique, such as PCA, to view the data in a multivariate and time-varying space. The results of such an analysis of the BATS and LSM realizations, together with the ECMWF analyses for comparison, are shown in Figure 3.

There is no a priori method to determine the significance of PCA loadings (Fig. 3a and b). An arbitrary significance level of an absolute value greater than 0.6 is suggested. Loadings falling below this are less well-correlated with the eigenvector variance, and hence less valuable for comparison. The score values (Fig. 3c and d) may be interpreted

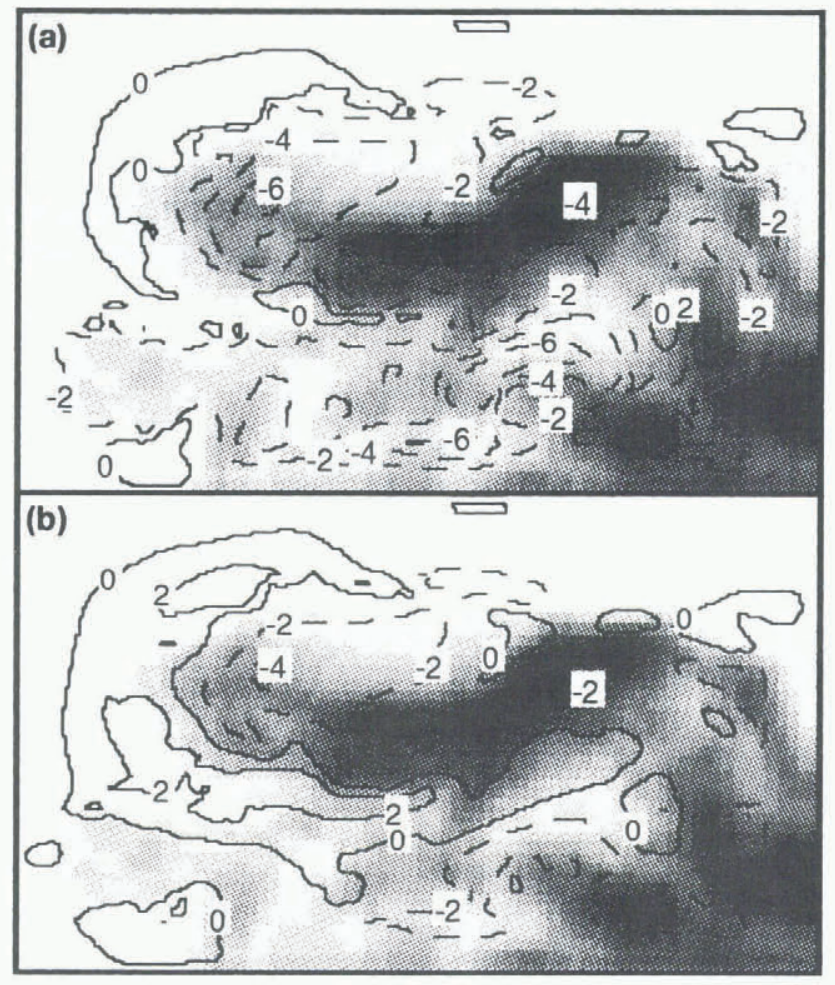

Fig. 2. Surface-air temperature biases for the (a) BATS and (b) LSM simulations compared to ECMWFanalyses in degrees $K$.
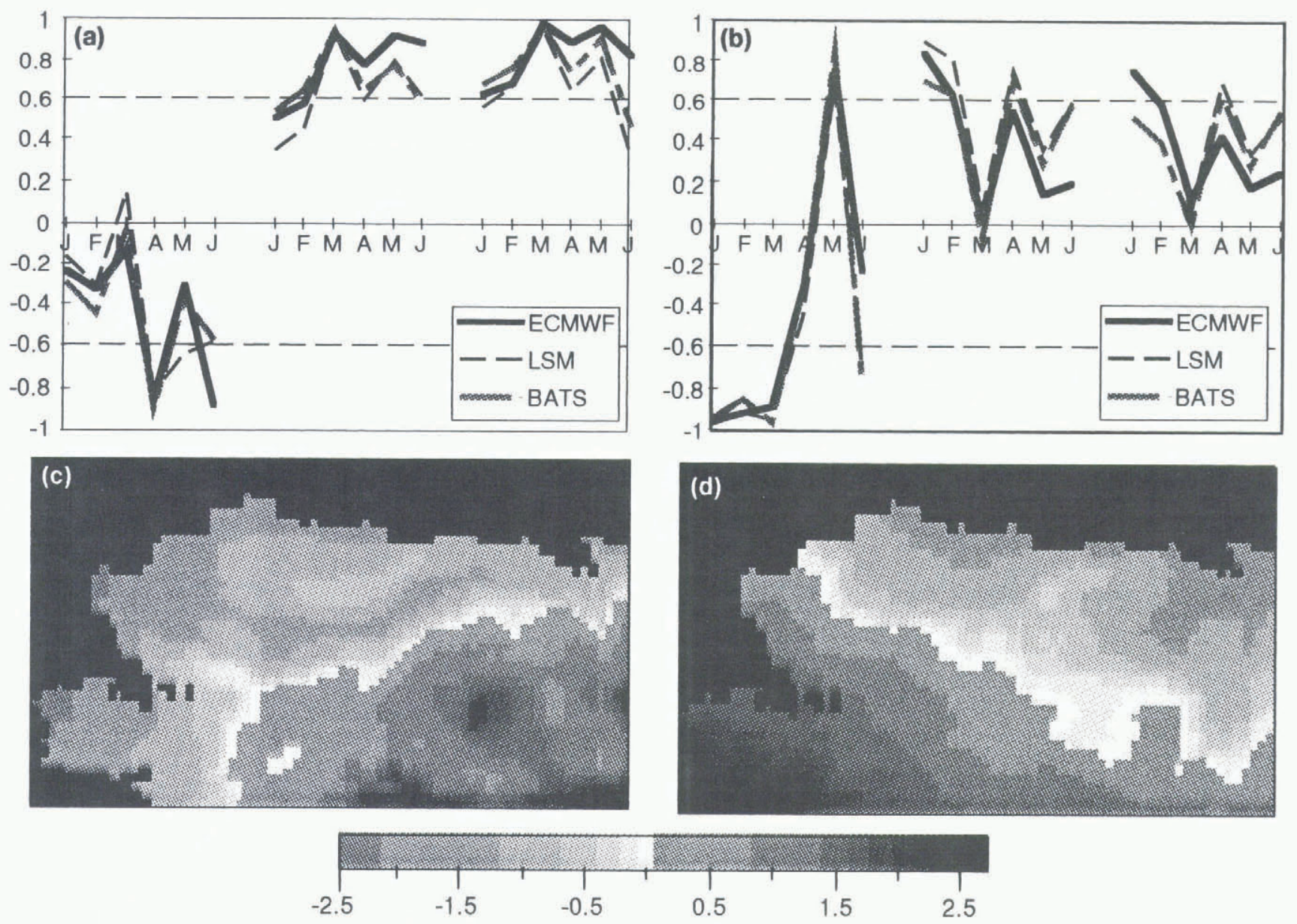

Fig. 3. Component loading patterns for ( a) eigenvector 1 ("summer/continental" paltern) and (b) eigenvector 2 ("winter/maritime" pattern ); from left to right, the patterns prepresent SLP, SFT and SFQ. The corresponding component scores from the LSM simulation are $(c)$ eigenvector 1 and $(d)$ eigenvector 2. 
as standard deviations from a pattern mean. The greater the score value, the more that gridcell demonstrates similarity to the loading values. For example, the January SFT loading is highly positive $(>0.6)$ and where the scores are also high $(>1.0)$ then those gridcells are "warm". Similarly, the January SLP loading is strongly negative $(<-0.6)$, and positive scores indicate regions of low pressure and negative scores indicate high-pressure areas.

The higher ECMWF loadings indicate a greater coherence of structure due to the lower resolution of the analyses, compared to the mesoscale structure that is possible in the ARCSyM results. The LSM and BATS loadings do not change through the spring transition as strongly or monotonically as the observational analyses. LSM, while following BATS in temperature, has a tendency in the moisture fields to exhibit more fluctuation between the summer and winter regimes. The scores associated with these loadings (representing the spatial variance) are shown in Figure $3 \mathrm{c}$ for the LSM simulation. The first eigenvector indeed represents a "summer/continental" pattern, primarily in temperature and moisture, with a thermal low centred over the southeastern part of the domain, associated with high temperatures and moisture in the boundary layer. This seasonal pattern shown in the loadings is interrupted somewhat in April and June, where loadings on the eigenvector drop below the 0.6 threshold; in both months, anomalous cyclone activity created a situation closer to the winter pattern described in eigenvector two below. This is reflected in the concomitant rise in the eigenvector two loading values on SFT and SFQ in these months. Regardless of this anomaly, the seasonal transition beginning in March remains evident, and would most likely be enhanced with a full annual cycle where more winter months are included in the analysis.

The second eigenvector loadings demonstrate a "winter/ maritime" pattern, primarily in SLP (Fig. 3b). Temperature also exhibits some of this winter pattern in the ARCSyM simulations, but the BATS moisture is less coherent with this pattern. Characteristic of this "winter/maritime pattern" are strong Aleutian and Bering Sea cyclonic activities combined with a zonal-temperature and moisture structure (Fig. 3d).

Major shortcomings of the use of ECMWF analyses to verify model ability include spatial resolution and the limited-input database, due to the operational nature of the analyses. By comparing the model realizations directly to station data, a more detailed picture of model performance can be developed. There are four stations performing regular soundings within the computational model domain. A selection of results from the Kotzebue station (indicated in Fig. 1) is shown in Figure 4.

Figure 4a shows the temperature biases between the BATS and LSM simulations throughout the six-month period at Kotzebue, for all vertical levels. Looking at the surface first, BATS is cooler in winter (January-February), and warmer in spring and summer, with greatest warming in April (a crucial transition period). The differences between the two runs extend strongly through the boundary layer and weakens to $<1^{\circ} \mathrm{C}$ above the low-level clouds (Fig. 4b), although there is also some mid-level bias (BATS warmer) in June. While cloudiness at all levels is not system-
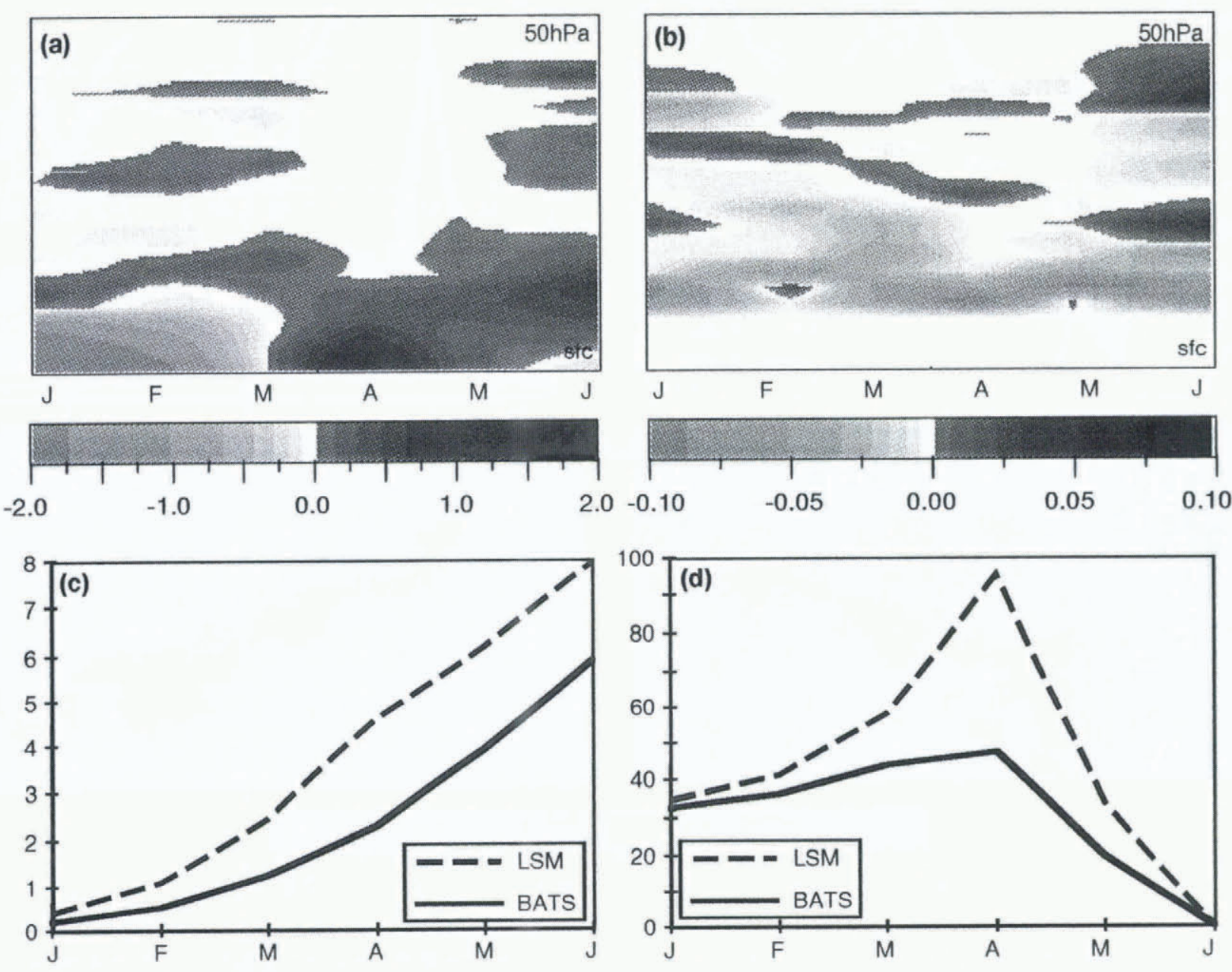

Fig. 4. Time series of BATS and LSM modelled quantities at Kotzebue: (a) air temperature differences (BATS-LSM); (b) cloud cover differences (BATS-LSM); (c) precipitation and (d) snow cover. 
atically greater in LSM, the low-level cloud is generally greater, and the total cloud over the column is also greater, until the month of June. The impact of the increased lowlevel cloud in LSM changes as the shortwave radiation increases from the winter zero minimum. In winter, with less low cloud in the BATS simulation, there is greater longwave cooling, with no incoming shortwave radiation (not shown). Moving into spring brings greater shortwave radiation, allowing warming of the surface in the BATS run, but not in the LSM run where there is greater low-level cloud cover. The precipitation (Fig. 4c) is larger in LSM in all months, with the difference increasing towards summer.

The warmer temperatures and lower precipitation in BATS allow earlier snowmelt (Fig. 4d). Because of the generally drier nature of the BATS run, this is reflected in smaller moisture fluxes from the surface (not shown) in LSM the surface latent heat flux is non-zero in winter, and continues to increase through spring as the BATS flux decreases.

There are two aspects of model formulation that are important here. Firstly, LSM allows for greater "surface pooling" of moisture by a delayed infiltration in the upper layer. Thus, there is more available moisture in the LSM package than in the BATS package allowing greater latent heat fluxes from the surface. Secondly, LSM includes a parameterization of snow albedo that depends on snow-grain size and has the effect that snow albedo decreases with increasing temperature. The effect of this can be seen in the more rapid rate of snowmelt in the ARCSyM-LSM simulation, which means that even with greater snow depth and lower temperatures, snow-free conditions are reached at about the same time. This behaviour is reflected in varying degrees at the other stations (Fairbanks, Nome and Barrow), and suggests that the hydrological cycle is crucial in controlling the differences between the BATS and LSM models.

\section{CONGLUSION}

This study used three methods to compare the behaviour in high latitudes of two land-surface vegetation models within a regional climate model. These methods included simple comparisons of model-output fields with observational analyses, a multivariate analysis using PCA, and direct comparison with station soundings. The use of the multivariate PCA provided an overview of the seasonal cycle, showing the transition between the dominant winter and summer patterns. The PCA also guided use of station data, and can indicate how representative behaviour at a single point is over a greater region.

It was found that the representation of the hydrological cycle is a crucial component in the accurate simulation of the Arctic boundary layer. In particular, the interactions between the seasonal cycles of incoming shortwave radiation, boundary-layer cloudiness and snow cover are extremely important and yet to be fully characterized.

Comparisons of this sort provide a valuable measure of land-surface model performance. BATS and LSM are widely used in GCMs and can potentially produce different impacts and strong feedbacks in the boundary layer, which will vary with latitude. Thus, it is vital to characterize fully the potential biases in different regions resulting from the choice of specific model components.

\section{REFERENCES}

Bonan, G. B. 1995. Land atmosphere $\mathrm{CO}_{2}$ exchange simulated by a land surface process model coupled to an atmospheric general circulation model. J. Geophys. Res., 100 D12, 2817-2831.

Bromwich, D. H., B. Chen and X. Pan. 1995. Intercomparison of simulated polar climates by global climate models. In Fourth Conference on Polar Meteorology and Oceanography, Dallas, Texas. Proceedings 79. Boston, MA, American Meteorological Society, 14-19.

Chapman, W. L. and J. E. Walsh. 1993. Recent variations of sea ice and air temperature in high latitudes. Bull. Am. Meteorol. Soc. 74 1),33-47.

Dickinson, R. E., A. Henderson-Sellers and P.J. Kennedy. 1993. Biosphere Atmosphere Transfer Scheme (B.ITS) version IE as coupled to the NCAR Community Climate Model. Boulder, CO, National Center for Atmospheric Research. NCAR Technical Note TN-387+STR.

Giorgi, F., M. R. Marinucci and G. T. Bates. 1993. Development of a second generation regional climate model (RegCM2. Part I. Boundary layer and radiative transfer processes. Mon. Weather Rev., 121 (10, 27942813.

Lynch, A. H.. W. L. Chapman, J. E. Walsh and G. Weller. 1995. Development of a regional climate model of the western Arctic. F. Climate, 8 6), 1555-1570.

McGinnis, D. L. and R. G. Crane. 1994. A multivariate analysis of Aretic climate in GCMs. J. Climate, 7 8, 1240-1250.

Maslanik, J. A., M. C. Serreze and R. G. Barry. 1996. Recent decreases in Arctic summer ice cover and linkages to atmospheric circulation anomalies. Geophys. Res. Lett., 23 (13), 1677-1680.

Osterkamp, T. E. and A. H. Lachenbruch. 1990. Thermal regime of permafrost in Alaska and the predicted global warming. ASCE. J. Cold Reg. Eng., 4 1), $38-42$.

Osterkamp, T. E., T. Zhang and V. E. Romanovsky. 1994. Evidence for a cyclic variation of permafrost temperatures in northern Alaska. Permafrost Periglacial Proc., 5 3).137-144.

Overland, J. E. and R. W. Priesendorfer. 1982. A significance test for principal components applied to a cyclone climatology. Mon. Weather Rer., $110(1), 1-4$.

Parkinson, C. L. and W. M. Washington. 1979. A large-scale numerical model of sea ice. .. Geophys. Res., 84 Cil, 311-337.

Shea, D. J., K. E. Trenberth and R. W. Reynolds. 1992. A global monthly sea surface temperature climatology. J. Climate, 5 9, 987-1001. 